\title{
Waveform Impact on Wireless Power Transfer Efficiency using Low-Power Harvesting Devices
}

\author{
Janis Eidaks* (Ph. D. Student, Institute of Radioelectronics, Riga Technical University, Riga, Latvia), \\ Anna Litvinenko (Senior Researcher, Institute of Radioelectronics, Riga Technical University, Riga, Latvia), \\ Arturs Aboltins (Senior Researcher, Institute of Radioelectronics, Riga Technical University, Riga, Latvia), \\ Dmitrijs Pikulins (Senior Researcher, Institute of Radioelectronics, Riga Technical University, Riga, Latvia)
}

\begin{abstract}
The paper addresses the impact of peak-to-average power ratio (PAPR) and spectrum of the waveform, as well as load resistance on the performance of low-power harvesting device in a real-life wireless power transfer (WPT) scenario. In the current study, a combination of the classic voltage doubler circuit for RFDC conversion and premanufactured device for DC-DC conversion is used. For the investigation of conversion efficiency and harvesting device performance, three types of waveforms are used: single tone, multicarrier signals with low PAPR and multicarrier signal with high PAPR. In order to generate highPAPR signal, subcarriers with the same amplitude and phase are summed, whereas for generation of low PAPR signal the phases of the subcarriers are chosen pseudo-randomly. Over-the-air transmission in $865 \mathrm{MHz}$ ISM band is made using directional antennas and all multicarrier waveforms have equal $5 \mathrm{MHz}$ bandwidth. To evaluate the performance of harvesting device and conversion efficiency, the average voltages at the input and output of the RF-DC converter as well as at the output of the DC-DC converter with corresponding input and load impedance are measured. The experiments have shown that the employed multicarrier signals can greatly improve the performance of harvesting device during WPT under certain conditions, which are discussed in the paper.
\end{abstract}

Keywords - Energy harvesting, DC-DC power converters, Internet of Things, low-power electronics, peak-to-average power ratio, radio frequency, wireless power transmission, wireless sensor networks.

\section{INTRODUCTION}

Extensive development of new wireless communication technologies in conjunction with the rapid evolution of microelectronics, leading to the highly reduced power consumption of embedded systems, has allowed for the widespread implementation of wireless sensor networks. This, consecutively, has formed the basis for the integration of Internet of Things (IoT) technologies into agriculture, transportation, healthcare and other areas.

One of the challenges on the way to the robust exploitation of sensor networks is to ensure the completely autonomous operation of nodes without the need to replace or manually recharge the batteries. One of the possible solutions to the problem is the use of radio frequency (RF) electromagnetic radiation for simultaneous sensor node powering and information transfer [1]. A widely used signal waveform for WPT is single-carrier transmission, due to multipath propagation inherent for in-door, and city application certain frequencies could be sufficiently attenuated, which can lead to WPT performance degradation. Thus, multi-tone signals potentially have advantage in a multi-path propagation case [2].

Furthermore, multipath propagation allows using timereversal for delivery of the energy to the intended receiver only [3]. In that case, all the available multipaths serve as virtual antennas.

Only few studies considered the methodology of optimal waveform design for the most efficient RF WPT. It has been shown theoretically in [4], [5] that the utilisation of broadband multi-tone signals with a high peak-to-average ratio (PAPR) can provide a significant boost in power transfer and conversion efficiency in comparison with traditional single-tone sinewaves. The problems of frequency-selective radio channel and non-linear receiver were addressed in [6], where the proposed analytical model of rectenna allowed for the design of new multi-tone waveforms. Another team of researchers [7] developed the state-of-the-art simplified methodology for the design of channel-adaptive WPT waveforms. In research [8], the problem of waveform design in WPT system with multiple receivers was addressed, whereas in paper [9] a complete model of WPT system with steerable energy beams between beacon and sensor nodes was proposed.

An increase in the number of in-phase subcarriers could potentially lead to the increase in the RF-to-DC conversion efficiency. However, research [10] demonstrates that a too high PAPR level in the experimental setup enhances degradation of power transfer efficiency. Moreover, these results have also been used for the next research [11], where authors show that the optimal resistance of the load depends on the PAPR level of the received RF signal. The research [12] gave theoretical simulations and calculations of the power conversion efficiency representing the sensitivity of the power conversion efficiency to the PAPR level (number of subcarriers summed in phase) of the employed RF signal.

In turn, our experimental study results given in [13] and [14] present advantages of an optimal PAPR level of multicarrier signal where subcarriers are summed in-phase for certain load resistance and input power levels. However, impact of load impedance in the case of low PAPR multicarrier signal, where specially selected phases for subcarriers with the same

\footnotetext{
* Corresponding author

E-mail: janis.eidaks@rtu.lv
} 
amplitude are used, still has not been sufficiently investigated. Moreover, the influence of the boost converter employment on the harvesting performance is also the field of interest for the application in WPT.

The current research is dedicated to the experimental study of influence of the PAPR level and the spectral characteristics of the multi-carrier signal, as well as load resistance on the WPT performance of low-power harvesting device consisting of a classic voltage doubler circuit for RF-DC conversion and a DCDC boost converter. The comparison of the experimentally obtained results with theoretical ones given in [12], where impact of the load resistance and PAPR level on the RF-DC conversion performance are studied via simulation, is presented in conclusion part of the research. Thus, the paper consists of four sections, where the employed signals for WPT and their properties are discussed in part A of Section II; the design of low-power harvesting device is given in part B of Section II, while methodology and measurement performance are explained in part C of Section II. In turn, results of the experimental study are shown in Section III and, finally, conclusion part with generalisations of the obtained results is presented in Section IV.

\section{EXPERIMENTAL SETUP}

The current section presents the methodology of the research including properties of tested signals, the design of the lowpower harvester and work performance, employed experimental setup for wireless power transfer and RF-DC conversion testing modes.

\section{A. Signals and their Properties}

For evaluation of the signal impact on RF-DC conversion and efficiency in WPT, three types of signals were selected: multitone signal with high PAPR level, multitone signal with a low PAPR level as well as a single tone signal. The number of carriers in the multitone signal varied from 4 to 16 carriers. The PAPR levels of all employed signals are shown in Table I. While these levels for multicarrier signals with a high PAPR increase along with sub-carrier number growth, in case of low PAPR signals they do not vary for all observed sub-carrier numbers. The single tone signal has the lowest PAPR value. The waveforms of all employed multicarrier signals are presented in Fig. 1. Moreover, all signals have the same average power.

For baseband signal generation, MATLAB/Simulink software was used, while for baseband signal transfer on carrier frequency and RF signal generation an SDR model USRP-B210 was used. A license-free sub-gigahertz band was chosen for RF signal generation.

In order to generate a multicarrier signal with a high PAPR, all subcarriers with the same amplitude were summed in phase. To generate signal with a low PAPR level, Zadoff-Chu sequences and an IFFT operation were employed and subcarriers with the same amplitude, but specially selected phases, were summed together. All performed multi-tone signals had the same spectrum bandwidth and carrier frequency: $5 \mathrm{MHz}$ and $865.5 \mathrm{MHz}$, correspondingly. The frequency of

single tone signal was equal to the centre frequency of the selected frequency band.

TABLE I

PAPR LEVEL OF EMPLOYED SIGNALS

\begin{tabular}{|l|c|c|}
\hline Signal waveforms & Subcarrier number & PAPR, dB \\
\hline \multirow{2}{*}{$\begin{array}{l}\text { High PAPR } \\
\text { signals }\end{array}$} & 4 & 10.09 \\
\cline { 2 - 3 } & 8 & 12.38 \\
\cline { 2 - 3 } Low PAPR signals & 16 & 15.84 \\
\cline { 2 - 3 } & 4 & 6.60 \\
\cline { 2 - 3 } & 8 & 6.06 \\
\hline Single tone & 16 & 5.99 \\
\hline
\end{tabular}

HPAPR
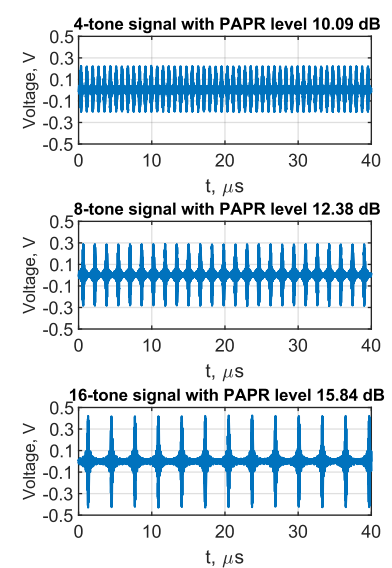

Fig. 1. Waveforms of the employed multicarrier signals with average power of $-10 \mathrm{dBm}$.

\section{B. Design of Low-Power Harvesting Device}

The selected harvesting device consists of simple RF-DC rectifier and DC-DC boost converter (see Fig. 2). The RF-DC rectifier is a voltage doubler with low-voltage drop Schottky diodes HSMS-285C. RF-DC voltage boosting to necessary voltage levels was performed by ultra-low-power boost converter BQ25504 from Texas Instruments [15]. In the latest revision, this converter started to function in "cold start" mode from $600 \mathrm{mV}$ input voltage till it charged the storage capacitor to $1.8 \mathrm{~V}$. Then it entered "normal mode", where it could function with the minimal input voltage level of just $130 \mathrm{mV}$ in addition to providing a minimum level of power for the IC to operate. This boost chip allows setting different thresholds and hysteresis voltage levels for the output, as well as undervoltage and overvoltage levels.

The threshold levels can be adjusted for the use of rechargeable batteries or low-voltage supercapacitors in order to prevent energy storage element discharging below the threshold value or charging above the critical voltage value. The voltage threshold levels are set by external resistors. The use of a resistor with a high resistance value has been recommended to decrease the power consumption. In the current design, the external n-channel MOSFET transistor was used to connect the chip output to load, when the programmed output voltage levels were reached. This chip also employs the 
maximum power point tracking (MPPT) feature for harvesting maximum power; however, it can be set only manually and for one user selected input voltage ratio level relative to open circuit value. This MPPT feature is not self-adapting; therefore, careful consideration for the MPPT level must be made. According to the information given in the datasheet, the converter total efficiency is highly dependent on the selected output voltage level, as well as on the input voltage level.

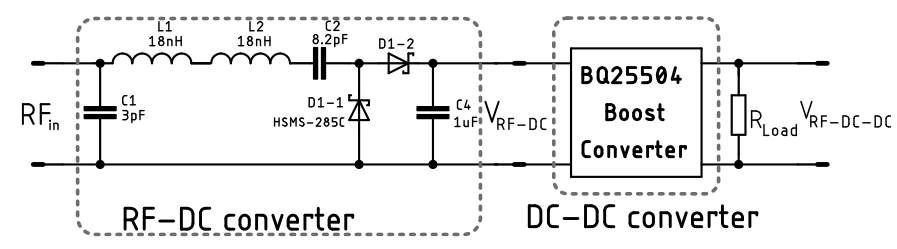

Fig. 2. Circuit of the employed low-power harvesting device with a DC-DC converter.

\section{Measurement Performance}

In the present study, the experiments were performed in multiple series to investigate the signal parameter impact on the RF-DC and DC-DC converter. The following experiments were conducted for the evaluation of the corresponding performance characteristic of converter performance:

1) the influence of the signal waveform on the efficiency of RF-to-DC conversion during wired energy transfer in case of variable load resistance at the RF-DC converter output - the setup of the measurement is presented in Fig. 3;

2) the influence of the signal waveform on the performance of the low-power harvesting device with an employed DC-DC converter in case of variable load at the DC-DC converter output - the setup of the measurement is given in Fig. 4;

3 ) the impact of the signal waveform on the performance of the WPT for the proposed low-power harvesting device consisting of an RF-DC converter in conjunction with a DC-DC converter - the setup of the measurement is shown in Fig. 5.

The multicarrier waveforms can be more advantageous due to its wideband nature in case of deep channel fading channel condition; this type of signal could provide less attenuation in comparison with the single tone signal when the channel fading happens in the transmitted signal frequency band. The advantage of high PAPR signal employment could be used to enable low-power harvesting devices via higher peaks of the signal. Waveforms with a high PAPR level were employed in the current RF-DC and the DC-DC conversion studies with different load impedances.

The maximum power of the RF-DC converter can be achieved, when the value of converter load impedance is the same as the output impedance.

In order to evaluate the first experiment, the rectifier output power for the signals with different carrier number and peak-toaverage power ratio levels were measured at different load values. In these experiments, equipment consisted of a PC with MATLAB/Simulink software, software defined radio USRP B210, RF-DC converter and voltmeter GDM-8246, the load resistance value at the RF-DC converter output varied from $1 \mathrm{k} \Omega$ to $200 \mathrm{k} \Omega$. The signal generated from the software defined radio USRP B210 was directly fed to RF-DC converter through a coaxial cable. The average power level of generated signals was $-10 \mathrm{dBm}$. The converter rectified voltage level on the output resistors was measured with the voltmeter GDM-8246. The converter open circuit voltage $\left(V_{\mathrm{RF}-\mathrm{DC} O \mathrm{OC}}\right)$ was also measured (see Table II). The test setup is shown in Fig. 3. The RF-DC converter output power level was calculated by squaring the output voltage $\mathrm{V}_{\mathrm{RF}-\mathrm{DC}}$ and dividing it by the resistance $R_{\mathrm{LOAD}}$.

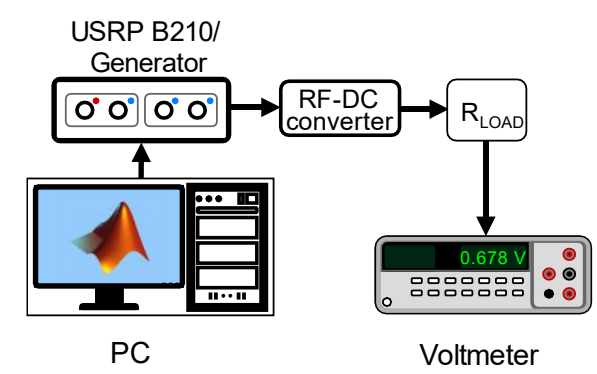

Fig. 3. Test setup for the RF-DC conversion measurements in wired testing mode.

In the second experiment, equipment consisted of a $\mathrm{PC}$ with MATLAB/Simulink software, SDR USRP B210, RF-DC and DC-DC converter, voltmeter GDM-8246, oscilloscope MSO5204B. The value of the RLOAD at the output of DC-DC converter also varied from $1 \mathrm{k} \Omega$ to $200 \mathrm{k} \Omega$. The signal generated from the USRP B210 was directly fed to RF-DC converter through a coaxial cable. The output of RF-DC converter was connected to the input of DC-DC converter. The average power level of generated signals was $-10 \mathrm{dBm}$. The test setup of this experiment is shown in Fig. 4. The DC-DC converter is required to boost the level of rectified voltage to a certain voltage level for the employed wireless sensor network node (e.g., LoRa) or other low power solution.

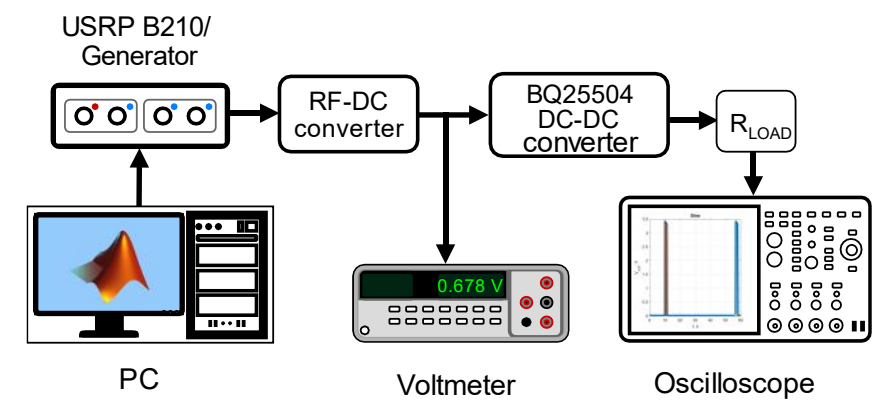

Fig. 4. Test setup for the RF-DC-DC conversion measurements in wired testing mode.

In these experiments, the DC-DC converter had a $4700 \mu \mathrm{F}$ storage capacitor, which was used to store the energy and supply the voltage to the load resistors when the programmed voltage levels were reached. The output voltage varied in range from $3.4 \mathrm{~V}$ to $3.3 \mathrm{~V}$ when the capacitor was discharging. In order to precisely deliver the specified voltage to the load, external n-channel MOSFET transistor was used and the control signal from the converter was used to switch the transistor. This boost converter allows using MPPT feature, where the boost converter indirectly modulates the input impedance of the main boost charger by regulating the charger input voltage set by 
resistor divider to VREF_SAMP input relative to an opencircuit voltage level.

In order to choose the most beneficial MPPT point, for the used signal waveforms, the RF-DC converter performance was evaluated at different output voltage levels by using different values of $R_{\mathrm{LOAD}}$. The optimal RF-DC converter output voltage in respect to the open circuit voltage for the maximum power was calculated (see Fig. 6, where the $\mathrm{x}$ axis shows the rectifier output voltage against the open circuit voltage in percent, $y$ axis shows the harvested power level).

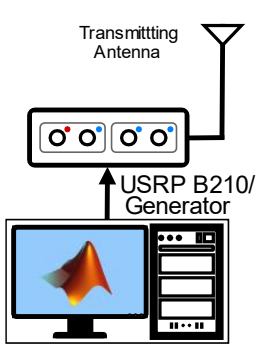

PC

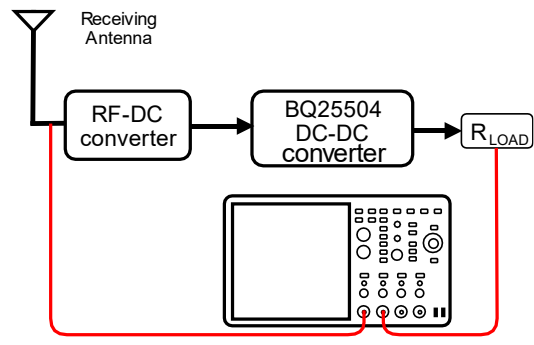

Oscilloscope
Fig. 5. Test setup for WPT performance measurement using the RF-DC-DC conversion.

As it can be concluded from Fig. 6, the RF-DC rectifier harvests the maximum power, when the RF-DC converter output voltage is at $45-50 \%$ of open circuit voltage for all the selected signal waveforms and number of carriers. Therefore, the MPPT level was set to $50 \%$ of input open circuit voltage.

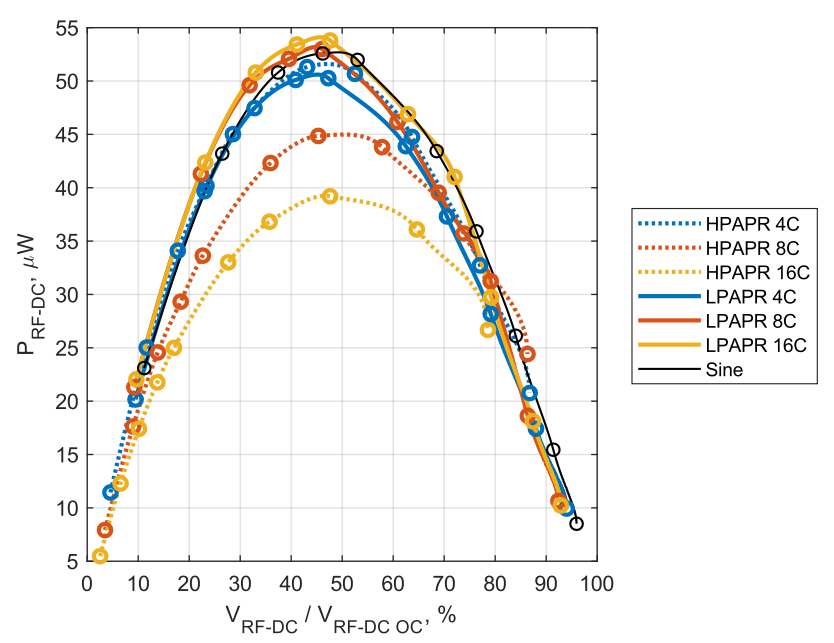

Fig. 6. RF-DC conversion output power levels for different MPPT levels.

During the measurements where RF-DC rectifier was operating with the DC-DC converter, the actual RF-DC converter voltage level was assessed to determine if the MPPT worked as intended. In the experiments where the RF signal is rectified and boosted using DC-DC converter, it is shown in Fig. 11, that the DC-DC converters load impudence value has little impact on the DC-DC converter efficiency. Therefore, in the following experiments the DC-DC converter performance was measured only at one impedance value, $10 \mathrm{k} \Omega$.

The measured voltages on RF-DC converter open circuit load, as well as with DC-DC converter as load impedance for different signals and carriers are shown in Table II. The results in Table II for the RF-DC with DC-DC converter were also measured with one $10 \mathrm{k} \Omega$ load value at the DC-DC converter output.

As shown in Table II, high PAPR signal waveforms provide the highest open circuit voltage; it varies for different number of carriers - increases as the number of carriers increases. For the low PAPR signal, the rectifier open circuit voltage fluctuates, but stays around the same value. The rectifier open circuit voltage is $14 \%$ higher in case of low PAPR signal waveforms compared to the single tone signal.

TABLE II

RF-DC CONVERTER OUTPUT VOLTAGE WITH DC-DC CONVERTER AND OPEN CIRCUIT OUTPUT

\begin{tabular}{|l|c|c|c|}
\hline \multirow{2}{*}{$\begin{array}{l}\text { Signal } \\
\text { waveforms }\end{array}$} & $\begin{array}{c}\text { ONLY RF-DC } \\
\text { converter }\end{array}$ & \multicolumn{2}{|c|}{$\begin{array}{c}\text { RF-DC } \\
\text { converter with DC-DC converter }\end{array}$} \\
\cline { 2 - 4 } Single tone & $V_{\mathrm{RF}-\mathrm{DC} O \mathrm{OC}}, \mathrm{V}$ & $V_{\mathrm{RF}-\mathrm{DC}}, \mathrm{V}$ & MPPT level \\
\hline HPAPR 4C & 1.36 & 0.678 & $49.85 \%$ \\
\hline HPAPR 8C & 2.35 & 1.01 & $42.97 \%$ \\
\hline HPAPR 16C & 2.65 & 1.07 & $40.38 \%$ \\
\hline LPAPR 4C & 1.5 & 1.05 & $35.71 \%$ \\
\hline LPAPR 8C & 1.58 & 0.774 & $51.33 \%$ \\
\hline LPAPR 16C & 1.54 & 0.774 & $48.99 \%$ \\
\hline
\end{tabular}

After setting the MPPT point of the DC-DC converter to $50 \%$, the RF-DC converter output voltage was measured again with the DC-DC converter as a load. For the signals with a low PAPR level and a single tone signal, the MPPT tracking works as intended. However, for the signals with a high PAPR level, the actual MPPT point shifts to lower voltage. For the carriers summed in-phase, the increase in PAPR level decreases the set MPPT point level.
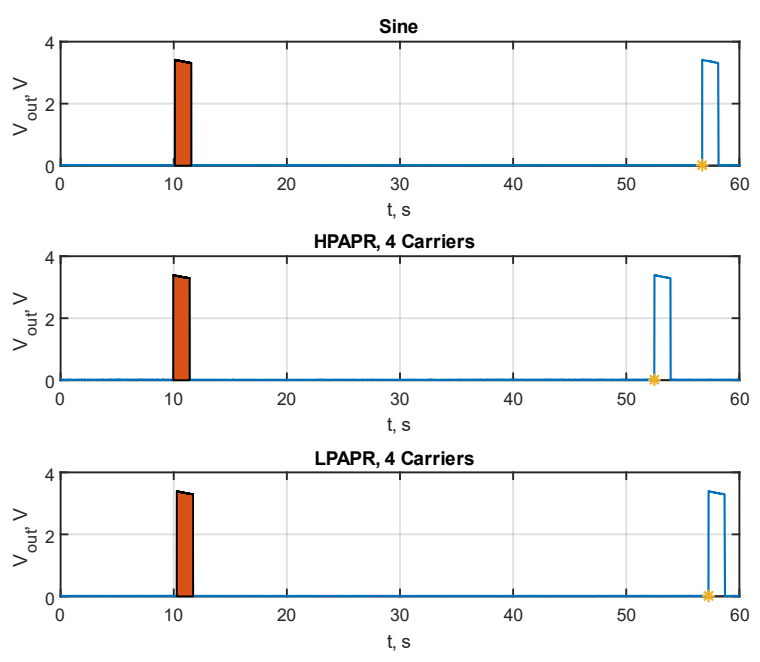

Fig. 7. DC-DC converter output voltage waveform with $10 \mathrm{k} \Omega$ load resistance with 4-subcarier multitone signals and sine signal. 
The output voltage of the DC-DC converter across the load resistors was measured with the oscilloscope; signal waveforms were saved and later processed in MATLAB. From the captured signal the average output power was calculated: one period was defined, signal was squared and divided by the load resistance, integrated over the selected period and then divided by the period. The example of the output voltage waveform of the DCDC converter is shown in Fig. 7. As it can be seen from the graph, the HPAPR signal for the RF-DC and DC-DC conversion allows charging the storage capacitor faster, thus delivering a higher average output power level.

The third experiment consisted of a PC with MATLAB/Simulink software, software defined radio USRP B210, RF-DC and DC-DC converter, MSO5204B oscilloscope, directional antennas LP0410. The value of the load resistance of DC-DC converter was set to $10 \mathrm{k} \Omega$. The signal generated from the software defined radio USRP B210 was fed to the transmitting antenna and the receiving antenna was connected to RF-DC converter. The RF-DC converter output was connected to the DC-DC converter input. The average power level of generated signals was $11.36 \mathrm{dBm}$. The test setup of this experiment is shown in Fig. 5.

\section{EXPERIMENTAL RESULTS}

The current section describes the experimental results of the influence of the waveform on the performance of a low-power harvesting device during WPT. The waveform impact has been studied for the standalone RF-DC converter and RF-DC converter in conjunction with a DC-DC converter. The wired and wireless energy transmission was studied in order to compare the influence of the waveform on RF-DC conversion in an ideal and real-life scenario.

\section{A. RF-to-DC Conversion in Case of Wired Power Transfer}

The impact of different signal waveforms and different PAPR levels on different parameters of the RF-DC converter with different load resistances in case of wired power transfer is shown in Figs. 8-10. The output voltages of RF-DC converter are shown in Fig. 8 and results depend on the load resistance. In case of high load resistance $>30 \mathrm{k} \Omega$ signals with a high PAPR level show higher voltage, while in case of lower resistance higher voltage is obtained for low PAPR and single tone signals. Besides, for load resistance less than $30 \mathrm{k} \Omega$ carrier the number for low PAPR signals does not influence results. For high PAPR signals there is a slight difference in voltage levels between different numbers of carriers for all observed resistance values. Moreover, the maximum obtained voltage is up to $2.3 \mathrm{~V}$ for 16 subcarriers summed in phase and $200 \mathrm{k} \Omega$ load, while single tone and low PAPR multi-tone signals provide only $1.3-1.5 \mathrm{~V}$ for the same load.

The influence of load resistance on the RF-to-DC converter power is presented in Fig. 9. The maximum power level above $50 \mu \mathrm{W}$ is obtained for single tone and multi-tone signals with a low PAPR level at $7.5-10 \mathrm{k} \Omega$ load resistance and for 4 subcarriers summed in-phase with a high PAPR level at 20-30 k $\Omega$ load resistance. The maximum power levels and corresponding load resistance differ for each subcarrier number of the high PAPR signal - the increase in the number of subcarriers leads to the growth of the resistance and decrease of power level maximum. For low PAPR signals, maximum power level and corresponding resistance are almost constant for all subcarrier numbers and mainly correspond to the single tone results. Current experimental results show the same trend as it has been demonstrated in simulation study [12], presenting higher RF-to-DC conversion performance of multi-tone signals with a high PAPR level in the case of high load resistance.

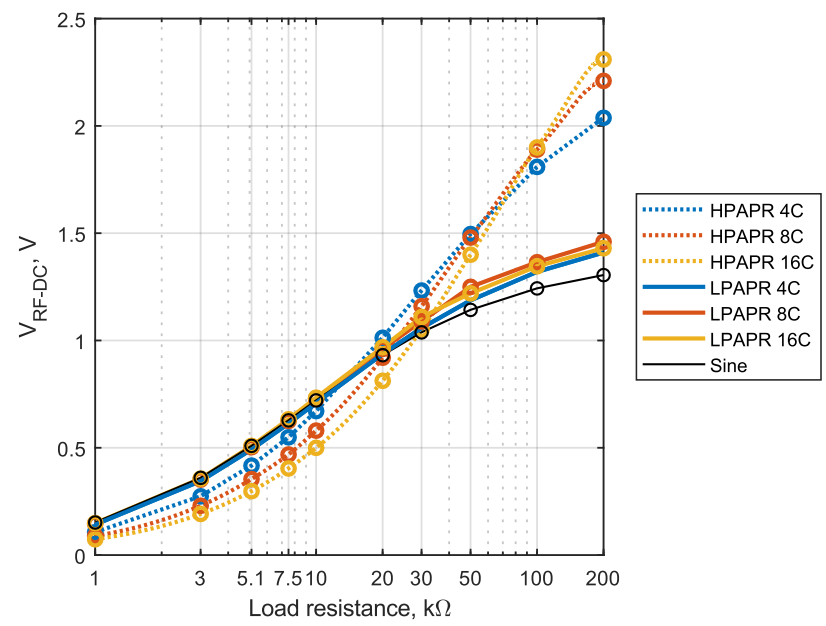

Fig. 8. RF-DC conversion output voltage for different load resistances and different signal waveforms.

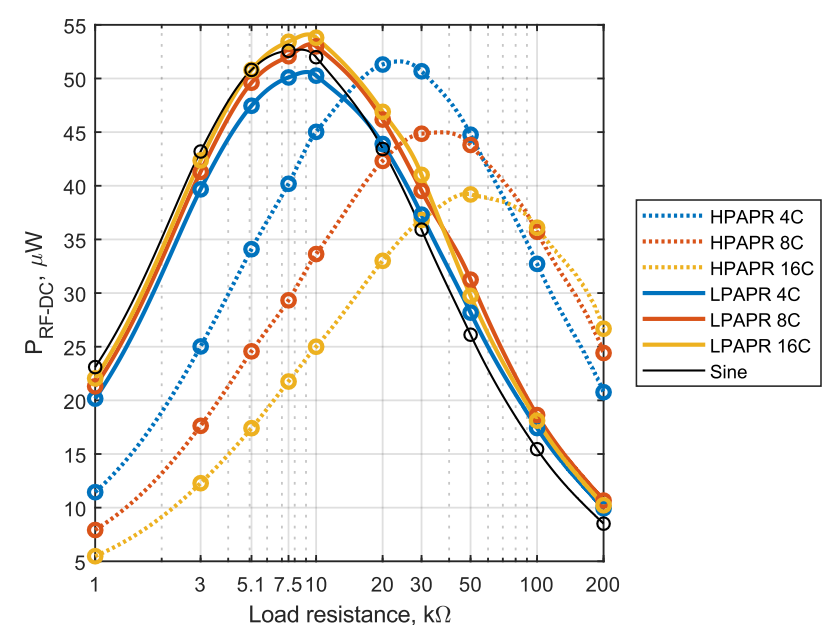

Fig. 9. RF-DC conversion output power levels for different load resistances and different signal waveforms.

Fig. 10 gives additional information about the efficiency of RF-to-DC conversion during wired power transfer. It shows that the maximum conversion efficiency higher than $50 \%$ is obtained for single tone and low PAPR multi-tone signals for the load of 7.5-10 k , as well as for 4 subcarriers summed inphase in the case of $20-30 \mathrm{k} \Omega$ load resistance. 


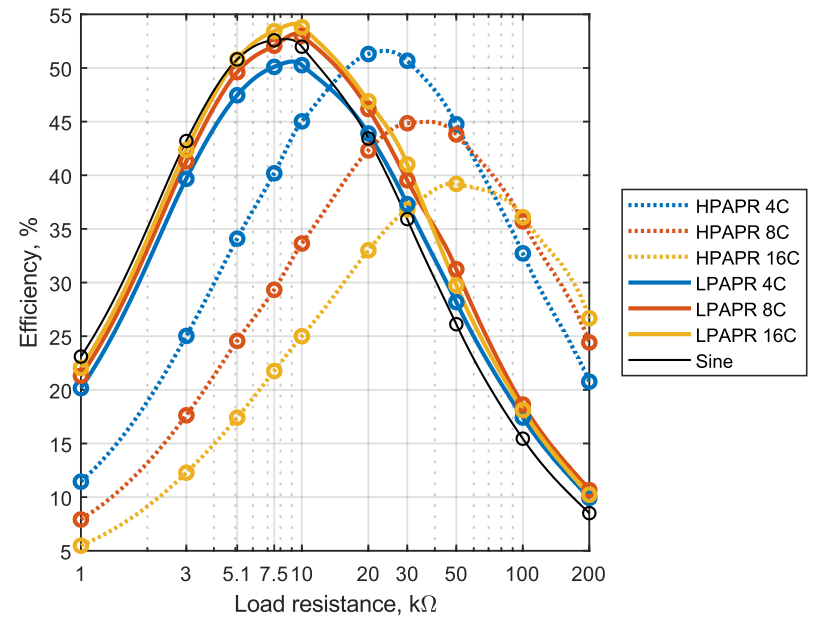

Fig. 10. RF-DC conversion efficiency for different load resistances and different signal waveforms.

\section{B. The Impact of DC-DC Converter on the Performance of Low-Power Harvesting Device}

The efficiency of the low-power harvesting device consisting of an RF-DC converter in conjunction with a DC-DC converter for different loads and signal waveforms is presented in Fig. 11. It is evident that the load resistance at DC-DC converter output does not influence the obtained power levels; the signal waveform has a high impact on the overall conversion performance of harvesting device. The highest efficiency above $35 \%$ - is shown by 4 subcarriers summed in-phase as well as 16 or 8 subcarriers with specially selected phases, while the efficiency level of $35 \%$ presents a single-tone signal. The lowest efficiency level, around $25 \%$, is obtained in case of 16 subcarriers summed in-phase. From results it can be seen that the number of subcarriers has direct impact on the conversion performance of the harvesting device with a DC-DC converter only in the case of high PAPR multicarrier signals.

The matching of the RF-DC converter was performed with the DC-DC converter for a single-tone signal, since the selected MPPT ratio sets up reasonably good matching (equivalent load) for the RF-DC converter. The RF-DC converter with an input power level of $-10 \mathrm{dBm}$ produced an output power of $52.59 \mu \mathrm{W}$ at $0.628 \mathrm{~V}$ for $7.5 \mathrm{k} \Omega$ load, when the DC-DC converter was loading the RF-DC circuit, the voltage at the output of RF-DC converter was kept at $0.678 \mathrm{~V}$, producing $52.45 \mu \mathrm{W}$ of power and thus working close to maximum power conditions.

\section{C.Performance of Low-Power Harvesting Device during WPT under Real-Life Scenario}

The results of performance evaluation of WPT measured for different distances in real-life conditions for the proposed lowpower harvesting device (consisting of the RF-DC and DC-DC converters) is shown in Fig. 12 as received power levels and converted power levels (levels at the output of harvesting device). The average received power in the antenna was measured using oscilloscope with $50 \Omega$ input, as the impedance of the antenna was also $50 \Omega$. The converted average power levels were calculated from the voltage across the load impedance at the output of the DC-DC converter.

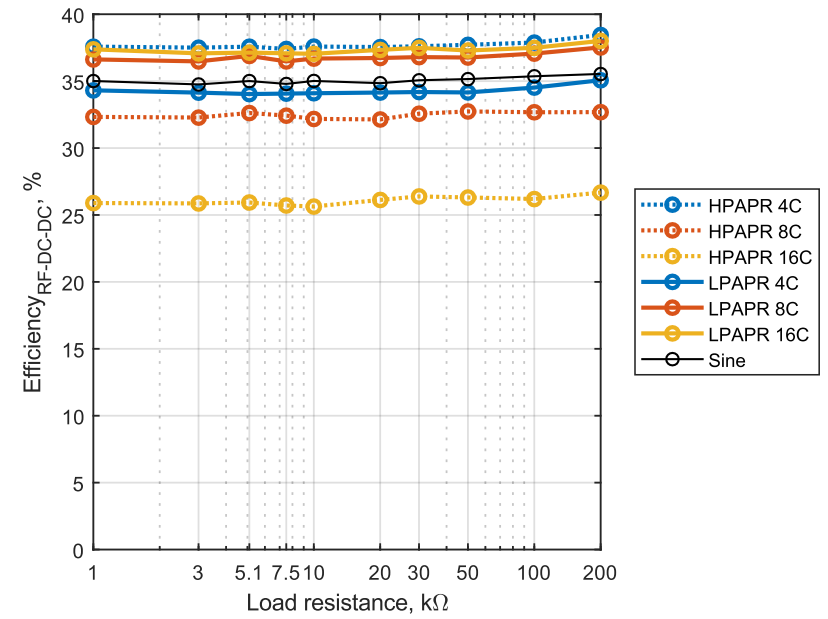

Fig. 11. RF-DC conversion efficiency of the harvesting device for different signal waveforms and different loads at the DC-DC converter output.

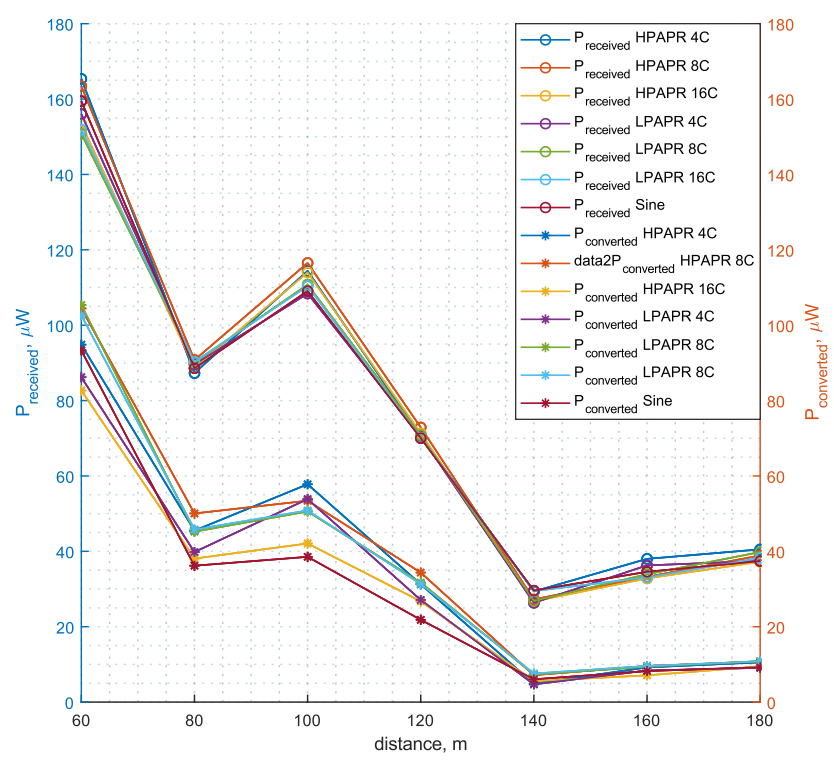

Fig. 12. Received average power level on antenna output and DC-DC converters average output power level.

As can be seen, the difference between the received power levels for different waveforms is not discernibly different, while the harvested and converted power levels depend not only on distance of energy transfer, but also on the waveform of the transmitted signal. Especially, this is evident for distance of $1 \mathrm{~m}$, where the difference between harvested power levels is around $9 \%$. In turn, for converted power levels it is around $50 \%$. The highest converted power level of $59 \mu \mathrm{W}$ is obtained for 4 carrier signals with a high PAPR level, a lower level shows 8 subcarriers summed in phase and multicarrier signals with a low PAPR level, while the single tone provides the lowest results of $37 \mu \mathrm{W}$.

The similarity in the received signal power levels could be explained by the same channel characteristic for carrier frequency of $865.5 \mathrm{MHz}$ and for bandwidth of $863-868 \mathrm{MHz}$ as a directional antenna was used during the experiment. 


\section{CONCLUSION}

The impact of signal spectral characteristics and PAPR level of the signal on the performance of the low-power harvesting device as well as the optimal load resistance during wireless power transfer (WPT) has been experimentally investigated. As the proposed low-power harvesting device consists of RF-DC rectifier and DC-DC boost converter, the efficiency of RF-toDC conversion and of the full harvesting device has been studied via controlled energy transfer and then validated under a real-life scenario in a typical office environment.

The RF-to-DC power conversion results show that the PAPR level and load resistance have a high impact on the conversion efficiency. Multicarrier signals with a low PAPR level show approximately the same results as the single tone, providing higher conversion efficiency at lower load resistance values. The multicarrier signal with high PAPR gives higher efficiency for higher values of load resistance. It has been shown that in case of a multi-tone signal with low PAPR the number of subcarriers does not have impact on the performance. However, in case of a high PAPR signal the growth of the number of subcarriers increases the value of optimal load resistance required to reach the maximal efficiency. The performed experiments justify the theoretical simulation results obtained in research [12].

If the RF-DC rectifier is used in conjunction with the DC-DC boost converter, the load resistance at the output of harvesting device does not impact the total performance of power conversion. However, the waveform itself has great influence on harvester performance. The same trend was also obtained during WPT study under a real-life scenario. Even if all signals are received with the same average power level, the conversion results differ up to $50 \%$.

The performed research complements previous studies with experimental results of the influence of the load resistance on RF-DC conversion efficiency, as well as for the case of connection of RF-DC rectifier and DC-DC boost converter. Moreover, the impact of multicarrier signals with different PAPR levels has been investigated showing that the use of multicarrier signals can improve the performance of harvesting device during WPT under certain conditions. The potential future research areas include the simulation and creation of the rectifier, which is matched to the HPAPR waveform. Yet another problem to overcome is miniaturisation of receiver antennas. One of the solutions would be the employment of miniature printed graphene antennas [16]. In this case, matching circuitry and waveforms must be elaborated even further.

\section{REFERENCES}

[1] B. Clerckx, R. Zhang, R. Schober, D. W. K. Ng, D. I. Kim, and H. V. Poor, "Fundamentals of Wireless Information and Power Transfer: From RF Energy Harvester Models to Signal and System Designs," IEEE J. Sel. Areas Commun., vol. 37, no. 1, pp. 4-33, Jan. 2018. https://doi.org/10.1109/JSAC.2018.2872615

[2] B. Clerckx and J. Kim, "On the Beneficial Roles of Fading and Transmit Diversity in Wireless Power Transfer with Nonlinear Energy Harvesting,' IEEE Trans. Wirel. Commun., vol. 17, no. 11, pp. 7731-7743, Nov. 2018 https://doi.org/10.1109/TWC.2018.2870377
[3] M.-L. Ku, Y. Han, H.-Q. Lai, Y. Chen, and K. J. R. Liu, "Power Waveforming: Wireless Power Transfer Beyond Time Reversal," IEEE Trans. Signal Process., vol. 64, no. 22, pp. 5819-5834, Nov. 2016. https://doi.org/10.1109/TSP.2016.2601283

[4] A. S. Boaventura and N. B. Carvalho, "Maximizing DC power in energy harvesting circuits using multisine excitation," in IEEE MTT-S International Microwave Symposium Digest, 2011, pp. 1-4. https://doi.org/10.1109/MWSYM.2011.5972612

[5] D. Belo, R. Fernandes, A. Collado, A. Georgiadis, and N. B. Carvalho, "Boosting the Efficiency," IEEE Microw. Mag., April, pp. 87-96, 2015.

[6] M. R. V. Moghadam, Y. Zeng, and R. Zhang, "Waveform optimization for radio-frequency wireless power transfer," IEEE Work. Signal Process. Adv. Wirel. Commun. SPAWC, vol. 2017-July, pp. 1-6, 2017. https://doi.org/10.1109/SPAWC.2017.8227719

[7] B. Clerckx and E. Bayguzina, "Low-Complexity Adaptive Multisine Waveform Design for Wireless Power Transfer," IEEE Antennas Wirel. Propag. Lett., vol. 16, pp. 2207-2210, 2017. https://doi.org/10.1109/LAWP.2017.2706944

[8] K.-W. Kim, H.-S. Lee, and J.-W. Lee, "Waveform Design for Fair Wireless Power Transfer With Multiple Energy Harvesting Devices," IEEE J. Sel. Areas Commun., vol. 37, no. 1, pp. 34-47, Jan. 2019. https://doi.org/10.1109/JSAC.2018.2872311

[9] K. W. Choi, L. Ginting, P. A. Rosyady, A. A. Aziz, and D. I. Kim, "Wireless-Powered Sensor Networks: How to Realize," IEEE Trans. Wirel. Commun., vol. 16, no. 1, pp. 221-234, Jan. 2017. https://doi.org/10.1109/TWC.2016.2621766

[10] A. Boaventura, N. B. Carvalho, and A. Georgiadis, "Unconventional Waveform Design for Wireless Power Transfer," in Nikoletseas S., Yang Y., Georgiadis A. (eds), Wireless Power Transfer Algorithms, Technologies and Applications in Ad Hoc Communication Networks. Cham: Springer International Publishing, 2016, pp. 137-159. https://doi.org/10.1007/978-3-319-46810-5_6

[11] F. Bolos, J. Blanco, A. Collado, and A. Georgiadis, "RF Energy Harvesting from Multi-Tone and Digitally Modulated Signals," IEEE Trans. Microw. Theory Tech., vol. 64, no. 6, pp. 1918-1927, Jun. 2016. https://doi.org/10.1109/TMTT.2016.2561923

[12] M. H. Ouda, P. Mitcheson, and B. Clerckx, "Optimal Operation of Multitone Waveforms in Low RF-Power Receivers," in 2018 IEEE Wireless Power Transfer Conference (WPTC), 2018, pp. 1-4. https://doi.org/10.1109/WPT.2018.8639426

[13] A. Litvinenko, J. Eidaks, and A. Aboltins, "Usage of Signals with a High PAPR Level for Efficient Wireless Power Transfer," in 2018 IEEE 6th Workshop on Advances in Information, Electronic and Electrical Engineering (AIEEE), 2018, pp. 1-5. https://doi.org/10.1109/AIEEE.2018.8592043

[14] A. Litvinenko, J. Eidaks, S. Tjukovs, D. Pikulins, and A. Aboltins, "Experimental Study of the Impact of Waveforms on the Efficiency of RF-to-DC Conversion Using a Classical Voltage Doubler Circuit,” in 2018 Advances in Wireless and Optical Communications (RTUWO), 2018, pp. 257-262. https://doi.org/10.1109/RTUWO.2018.8587907

[15] Texas Instruments, "BQ25504 ultra low-power boost converter with battery management for energy harvester applications." Datasheet, p. 39, 2014.

[16] V. Slegeryte, D. Belova-Ploniene, A. Katkevicius, and D. Plonis, "Microwave Devices with Graphene Layers: A Review," in Proceedings of 2019 IEEE Microwave Theory and Techniques in Wireless Communications, MTTW 2019, 2019, pp. 87-92. https://doi.org/10.1109/MTTW.2019.8897243

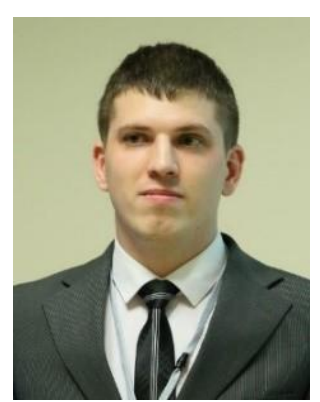

Janis Eidaks received his Bachelor degree from Riga Technical University, Riga, Latvia in 2015 and Master degree from Riga Technical University in 2017. He is currently a Doctoral student at the Department of Electronics and Telecommunication of Riga Technical University. His research interests include autonomous powering solution development. E-mail: janis.eidaks@rtu.lv

ORCID iD: https://orcid.org/0000-0002-89846621 


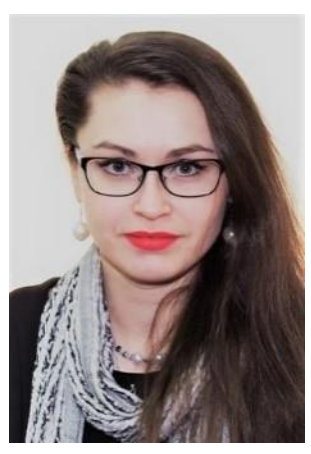

Anna Litvinenko has received Bachelor (2008), Master (2010) and Doctoral (2017) degrees in in Electronics from Riga Technical University. She is an Assistant Professor and a Senior Researcher at the Institute of Radioelectronics, Riga Technical University, Latvia. She has been an IEEE member since 2013 and Officer of IEEE Latvia section Membership Development since 2016. Moreover, Anna has been an expert and reporter of the European Commission since 2014, a member of RTU Constitutional Assembly since 2018, and chair of international Workshop on Microwave Theory and Techniques in Wireless Communications MTTW'2019. Her research interests include wireless sensor networks, IoT solutions, communication systems, signal processing.

E-mail: anna.litvinenko@rtu.lv

ORCID iD: https://orcid.org/0000-0003-4734-2182

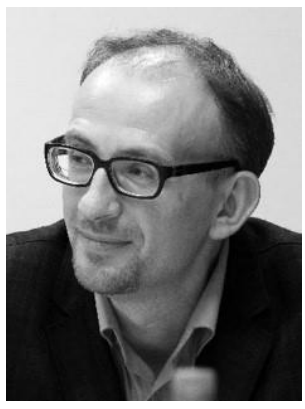

Arturs Aboltins holds Doctoral (2013) degree from Riga Technical University. Before receiving his Doctoral degree, he has held both technical and management positions in the Latvian industry for more than 10 years. He is a Senior Researcher at the Institute of Radioelectronics and an Associate Professor and Head of the Department of Electronics Fundamentals at RTU. His research interests include the application of unitary transforms and software-defined radio in communication systems. He is the founder and co-founder of several international scientific conferences such as Advances in Wireless and Optical Communications (RTUWO) and Workshop on Microwave Theory and Techniques in Wireless Communications (MTTW). He is a Chair of the IEEE Latvia Section COM/MTT/AP Joint Societies Chapter.

E-mail: arturs.aboltins_1@ @rtu.lv ORCID iD: http://orcid.org/0000-0001-6901-9787

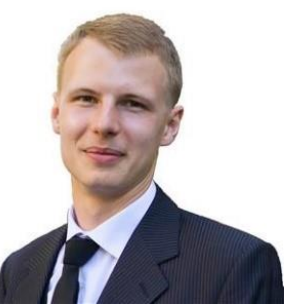

Dmitrijs Pikulins received his Doctoral (2012) degree in Electronics from Riga Technical University. He is an Associate Professor and a Senior Researcher at the Institute of Radioelectronics, Riga Technical University, Latvia. Moreover, Dmitrijs is an expert at the Latvian Academy of Sciences, a member of RTU

Constitutional Assembly and the Head of the Institute of Radioelectronics. His research interests include wireless sensor networks, IoT, nonlinear chaotic systems, power electronics.

E-mail: dmitrijs.pikulins@rtu.lv

ORCID iD: https://orcid.org/0000-0002-6768-6989 